\title{
ON THE EUCLIDEAN ALGORITHM IN QUADRATIC NUMBER FIELDS
}

\author{
H. ChATLAND
}

1. Introduction. Let $m$ be a square-free rational integer. The field $R\left(m^{1 / 2}\right)$ is said to be Euclidean or that the Euclidean algorithm exists in $R\left(m^{1 / 2}\right)$ if for integers $\alpha, \beta \neq 0 \subset R\left(m^{1 / 2}\right)$ there exists an integer $\gamma \subset R\left(m^{1 / 2}\right)$ such that

$$
|N(\alpha-\beta \gamma)|<|N(\beta)| \text {. }
$$

The problem of determining in what fields $R\left(m^{1 / 2}\right)$ the algorithm exists has been worked out except for $m$ equal to a prime of the form $24 n+1$ and greater than 97. In this paper it is shown that the Euclidean algorithm does not exist for $m=24 n+1>97$ except possibly for $m=193,241,313,337,457$, and 601 . The problem is not settled in these six cases.

2. Previous results. In order that a field be Euclidean the class number must be 1 . However, this condition is not sufficient for, as Dedekind pointed out [1 $]^{1}$, the field $R\left(-19^{1 / 2}\right)$ has class number 1 but is not Euclidean. L. E. Dickson [2] showed that for $m$ negative the Euclidean algorithm exists only if $m=-1,-2,-3,-7$, and -11 . For $m$ positive, the algorithm has been shown to exist for the following values of $m$ :

$$
2,3,5,6,7,11,13,17,19,21,29,33,37,41,57,73,97 .
$$

Except for the last two values in (1) the proofs have been obtained by O. Perron [3], A. Oppenheim [4], R. Remak [5], N. Hofreiter [6], and A. Berg [7]. It was pointed out by I. Schur [4, p. 351] that the algorithm does not exist for $m=47$. A. Oppenheim [4] proved that for $m=23$ and $m=53$ the algorithm does not exist. N. Hofreiter [8] proved non-existence for $m \equiv 14(\bmod 24)$ and [6] for $m=77$ and $m \equiv 21(\bmod 24), m>21$. E. Berg [7] and J. F. Keston [9] proved nonexistence for $m \neq \equiv 1(\bmod 4)$ except for the values listed in (1). Also, apart from (1) H. Behrbohm and L. Rédei [10] showed that the algorithm can exist only in the following three cases.

$$
\text { I. } m=p \equiv 13(\bmod 24) \text {, }
$$

Presented to the Society, June 19, 1948; received by the editors May 11, 1948, and in revised form, July 23, 1948.

${ }^{1}$ Numbers in brackets refer to the bibliography at the end of the paper. 
II. $\quad m=p \equiv 1(\bmod 8)$,

III. $m=p q$ with $p \equiv q \equiv 3(\bmod 8)$ or $p \equiv q \equiv 7(\bmod 8)$

where $p$ and $q$ are primes.

For sufficiently large $m, \mathrm{P}$. Erdös and Ch. Ko [11] proved that the algorithm cannot exist in cases I and II. H. Heilbronn [12] proved a similar result for case III. L. Schuster [13] showed that except for $m=33$ and 57 in case III the algorithm exists at most for $m \equiv 1$ $(\bmod 24)$. A. Brauer [14] proved that the algorithm cannot exist in case $I$ for $p>109$. There remained then in case $I$ only the values $m=61$ and $m=109$. L. Rédei [15] proved the non-existence of the algorithm for these two values. By an entirely different method L. K. Hua and W. T. Sheh [17] proved that the algorithm does not exist for $m=61$. L. Rédei [18] obtained the result in case III that if the algorithm exists then $m=3 q$. This result coupled with that of Schuster [13] completes case III in which the algorithm exists only for $m=21,33,57$. There remain those values of $m=p \equiv 1(\bmod 8)$. For $p=73,97, \mathrm{~L}$. Rédei [15] proved the existence of the algorithm and for $p$ of the form $24 n+17$ and greater than 41 the non-existence of the algorithm. The case $p=24 n+17$ and $p>41$ was also treated by L. Hua and S. Min [16] who left in doubt however $p=89,113$, and 137.

3. Present results. Recently H. Danveport [19] proved that the Euclidean algorithm does not exist for quadratic fields whose discriminants exceed $(128)^{2}$. In their paper, mentioned above, Erdös and Ko prove the following theorem:

THEOREM. For a prime $p$ of the form $4 n+1$, the Euclidean algorithm cannot exist in $R\left(p^{1 / 2}\right)$, if $p$ can be written in the form

$$
p=q_{1} m_{1}+q_{2} m_{2},
$$

where $m_{1}, m_{2}, q_{1}, q_{2}$ are all positive and quadratic non-residues $(\bmod p)$, and where the $q_{i}$ are odd primes which divide $q_{i} m_{i}$ to an odd power for $i=1,2$.

In this paper a representation of the form (2) is given for each prime of the form $24 n+1$ greater than 97 and less than $(128)^{2}$ except for $p=193,241,313,337,457$, and 601 . In the case of these last six it can be shown that no such representation exists. Hence for them no conclusion can be drawn, concerning the existence of the algorithm, by this method. 
Representations of primes $p=24 n+1$ in the form $p=q_{1} m_{1}+q_{2} m_{2}$

\begin{tabular}{|c|c|c|c|c|c|}
\hline $409=14 x$ & $19+11 \times$ & 13 & $3169=7 \times$ & $86+17 \times$ & 151 \\
\hline $433=7 \times$ & $29+10 x$ & 23 & $3217=5 \times$ & $87+13 x$ & 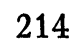 \\
\hline $577=14 X$ & $13+5 x$ & 79 & $3313=13 x$ & $45+11 \times$ & 248 \\
\hline $673=17 x$ & $15+19 x$ & 22 & $3361=11 \times$ & $68+13 \times$ & \\
\hline $769=23 x$ & $21+13 x$ & 22 & $3433=7 \times$ & $10+19 x$ & \\
\hline $937=5 \times$ & $7+41 \times$ & 22 & $3457=5 \times$ & $42+17 \times$ & \\
\hline $1009=11 X$ & $52+19 \times$ & 23 & $3529=13 \times$ & $57+17 \times$ & \\
\hline $1033=11 X$ & $13+89 x$ & 10 & $3673=5 \times$ & $13+11 x$ & \\
\hline $1129=17 \times$ & $19+31 \times$ & 26 & $3697=5 \times$ & $92+13 x$ & \\
\hline $1153=17 \times$ & $14+61 \times$ & 15 & $3769=17 \times$ & $14+11 \times$ & \\
\hline $1201=17 \times$ & $26+23 x$ & 33 & $3793=19 \times$ & $60+7 x$ & \\
\hline $1249=19 \times$ & $44+7 \times$ & 59 & $3889=11 \times$ & $38+13 \times$ & \\
\hline $1297=5 \times$ & $154+17 \times$ & 31 & $4057=29 \times$ & $20+19 x$ & \\
\hline $1321=17 \times$ & $39+47 \times$ & 14 & $4129=7 \times$ & $29+13 \times$ & 30 \\
\hline $1489=17 x$ & $39+59 \times$ & 14 & $4153=13 \times$ & $102+11 \times$ & \\
\hline $1609=19 x$ & $14+17 \times$ & 79 & $4177=11 \times$ & $45+7 \times$ & $\mathrm{J}$ \\
\hline $1657=7 \times$ & $45+61 \times$ & 22 & $4201=11 \times$ & $38+13 x$ & \\
\hline $1753=7 \times$ & $165+13 \times$ & 46 & $4273=5 \times$ & $58+7 \times$ & 50 \\
\hline $1777=5 \times$ & $21+19 x$ & 88 & $4297=5 \times$ & $19+11 x$ & \\
\hline $1801=19 \times$ & $78+11 \times$ & 29 & $4441=13 \times$ & $70+11 \times$ & 32 \\
\hline $1873=23 \times$ & $60+17 \times$ & 29 & $4513=19 x$ & $15+7 x$ & \\
\hline $1993=7 \times$ & $5+89 \times$ & 22 & $4561=17 \times$ & $110+13 x$ & 20 \\
\hline $2017=5 \times$ & $26+17 \times$ & 111 & $4657=5 \times$ & $46+19 x$ & \\
\hline $2089=7 \times$ & $190+11 \times$ & 69 & $4729=11 \times$ & $93+17 \times$ & 21 \\
\hline $2113=5 \times$ & $277+7 \times$ & 104 & $4801=23 \times$ & $21+17 \times$ & 2. \\
\hline $2137=13 \times$ & $129+5 x$ & 92 & $4969=7 \times$ & $152+11 \times$ & 35 \\
\hline $2161=23 \times$ & $70+19 \times$ & 29 & $4993=5 \times$ & $57+11 \times$ & 42 \\
\hline $2281=7 \times$ & $51+13 \times$ & 148 & $5113=5 \times$ & $93+7 \times$ & 60 \\
\hline $2377=5 \times$ & $62+13 \times$ & 159 & $5209=11 \times$ & $306+19 x$ & 9 \\
\hline $2473=5 \times$ & $31+19 x$ & 122 & $5233=5 \times$ & $17+11 \times$ & 468 \\
\hline $2521=11 \times$ & $53+17 \times$ & 114 & $5281=7 \times$ & $38+17 \times$ & 29 \\
\hline $2593=5 \times$ & $275+29 \times$ & 42 & $5449=7 \times$ & $95+13 \times$ & 36 \\
\hline $2617=7 \times$ & $122+41 \times$ & 43 & $5521=7 \times$ & $89+31 \times$ & 15 \\
\hline $2689=13 x$ & $46+17 \times$ & 123 & $5569=19 \times$ & $68+13 x$ & 329 \\
\hline $2713=11 \times$ & $153+5 \times$ & 206 & $5641=7 \times$ & $172+29 \times$ & 15 \\
\hline $2833=7 \times$ & $15+11 \times$ & 248 & $5689=17 \times$ & $132+13 \times$ & 26 \\
\hline $2857=5 \times$ & $138+11 \times$ & 197 & $5737=37 \times$ & $20+19 x$ & 26 \\
\hline $2953=5 \times$ & $42+13 \times$ & 211 & $5857=5 \times$ & $78+7 \times$ & 78 \\
\hline $3001=7 x$ & $65+19 x$ & 134 & $5881=31 \times$ & $13+11 \times$ & 49 \\
\hline $3049=11 \times$ & $94+13 \times$ & 155 & $5953=5 \times$ & $7+11 \times$ & 53 \\
\hline $3121=7 \times$ & $220+17 \times$ & 93 & $6073=5 \times$ & $29+19 x$ & 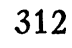 \\
\hline
\end{tabular}




$6121=7 \times \quad 87+13 \times 424$
$6217=17 \times \quad 15+11 \times 542$
$6257=5 \times \quad 56+43 \times 139$
$6337=5 \times \quad 61+13 \times 464$
$6361=17 \times \quad 21+19 \times 316$
$6481=13 \times 342+11 \times 185$
$6529=11 \times 141+19 \times 262$
$6553=11 \times 280+23 \times 151$
$6577=5 \times \quad 69+19 \times 328$
$6673=5 \times 308+29 \times 177$
$6793=7 \times 235+11 \times 468$
$6841=29 \times \quad 67+31 \times 158$
$6961=7 \times 104+23 \times 271$
$7057=13 \times \quad 80+11 \times 547$
$7129=7 \times 374+13 \times 347$
$7177=5 \times 38+17 \times 411$
$7297=7 \times \quad 15+29 \times 248$
$7321=7 \times 312+11 \times 467$
$7369=13 \times 201+29 \times 164$
$7393=23 \times \quad 29+19 \times 354$
$7417=5 \times 17+13 \times 564$
$7489=37 \times \quad 70+23 \times 213$
$7537=5 \times \quad 51+11 \times 662$
$7561=13 \times \quad 73+29 \times 228$
$7681=13 \times 102+31 \times 205$
$7753=5 \times 416+31 \times 183$
$7873=13 \times \quad 10+29 \times 267$
$7993=5 \times \quad 88+7 \times 1079$
$8017=19 \times \quad 90+17 \times 371$
$8089=17 \times 295+29 \times 106$
$8161=7 \times \quad 29+23 \times 346$
$8209=13 \times \quad 14+23 \times 349$
$8233=17 \times 165+23 \times 236$
$8329=31 \times 219+11 \times 140$
$8353=5 \times \quad 17+13 \times 636$
$8377=13 \times 135+11 \times 602$
$8521=13 \times \quad 31+11 \times 738$
$8641=7 \times 136+11 \times 699$
$8689=29 \times 195+37 \times 82$
$8713=5 \times 404+23 \times 291$
$8737=37 \times \quad 30+29 \times 263$
$8761=19 \times \quad 71+17 \times 436$

$$
\begin{aligned}
& 8929=13 \times 114+11 \times 677 \\
& 9001=13 \times 42+19 \times 445 \\
& 9049=11 \times 53+17 \times 498 \\
& 9241=17 \times 286+29 \times 151 \\
& 9337=5 \times 203+19 \times 438 \\
& 9433=13 \times 80+11 \times 763 \\
& 9601=13 \times 174+41 \times 179 \\
& 9649=41 \times 212+11 \times 87 \\
& 9697=11 \times 30+17 \times 551 \\
& 9721=11 \times 774+17 \times \quad 71 \\
& 9769=17 \times 390+43 \times 73 \\
& 9817=5 \times 42+13 \times 739 \\
& 10009=11 \times 105+19 \times 466 \\
& 10177=5 \times \quad 7+11 \times 922 \\
& 10273=5 \times 238+31 \times 293 \\
& 10321=7 \times \quad 41+29 \times 346 \\
& 10369=13 \times 207+11 \times 698 \\
& 10513=5 \times 28+11 \times 943 \\
& 10657=29 \times 353+7 \times 60 \\
& 10729=41 \times 53+23 \times 372 \\
& 10753=5 \times 351+11 \times 818 \\
& 10993=5 \times 156+7 \times 1459 \\
& 11113=5 \times 611+17 \times 474 \\
& 11161=7 \times 66+13 \times 823 \\
& 11257=17 \times 190+23 \times 349 \\
& 11329=11 \times 140+13 \times 753 \\
& 11353=7 \times 19+17 \times 660 \\
& 11497=7 \times 60+11 \times 1007 \\
& 11593=11 \times \quad 40+19 \times 587 \\
& 11617=13 \times 245+17 \times 496 \\
& 11689=7 \times 264+13 \times 757 \\
& 11833=7 \times 20+11 \times 1063 \\
& 11953=5 \times 22+13 \times 911 \\
& 12049=19 \times 156+23 \times 395 \\
& 12073=7 \times \quad 15+11 \times 1088 \\
& 12097=11 \times 120+13 \times 829 \\
& 12241=13 \times 126+23 \times 461 \\
& 12289=19 \times 165+23 \times 398 \\
& 12409=13 \times 63+19 \times 610 \\
& 12433=13 \times 160+17 \times 609 \\
& 12457=19 \times 330+23 \times 269 \\
& 12553=11 \times 230+13 \times 771
\end{aligned}
$$




$$
\begin{aligned}
& 12577=5 \times 126+13 \times 919 \\
& 12601=29 \times 132+31 \times 283 \\
& 12721=17 \times \quad 52+19 \times 623 \\
& 12841=23 \times \quad 86+17 \times 639 \\
& 12889=11 \times 195+17 \times 632 \\
& 13009=7 \times 23+11 \times 1168 \\
& 13033=5 \times 102+7 \times 1789 \\
& 13177=7 \times \quad 80+11 \times 1147 \\
& 13249=7 \times 13+17 \times 774 \\
& 13297=5 \times 153+13 \times 964 \\
& 13417=11 \times \quad 40+19 \times 683 \\
& 13441=29 \times 110+17 \times 603 \\
& 13513=5 \times 126+13 \times 991 \\
& 13537=5 \times 63+11 \times 1202 \\
& 13633=5 \times 569+31 \times 348 \\
& 13681=11 \times \quad 69+13 \times 994 \\
& 13729=17 \times 328+31 \times 263 \\
& 13873=11 \times \quad 78+19 \times 685 \\
& 13921=11 \times 118+13 \times 971 \\
& 14281=13 \times 124+41 \times 309 \\
& 14401=11 \times 145+19 \times 674 \\
& 14449=11 \times \quad 62+13 \times 1059
\end{aligned}
$$

\section{BIBLIOGRAPHY}

1. P. G. L. Dirichlet, Vorlesungen iber Zahlentheorie, ed. by R. Dirichlet, 4th ed., Braunschweig, 1894, p. 451.

2. L. E. Dickson, Algebren und ihre Zahlentheorie, Zürich and Leipzig, 1927, pp. 150-151.

3. O. Perron, Quadratische Zahlkörper mit Euklidischen Algorithmus, Math. Ann. vol. 107 (1932) pp. 489-495.

4. A. Oppenheim, Quadratic fields with and without Euclid's algorithm, Math. Ann. vol. 109 (1934) pp. 349-352.

5. R. Remak, Über den Euklidischen Algorithmus in reellquadratischen Zahlkörpern, Jber. Deutschen Math. Verein. vol. 44 (1934) pp. 238-250.

6. N. Hofreiter, Quadratische Körper mit und ohne Euklidischen Algorithmus, Monatshefte für Mathematik und Physik vol. 42 (1935) pp. 397-400.

7. E. Berg, Über die Existenz eines Euklidischen Algorithmus in quadratischen Zahlkörpern, Kungl. Fysiografiska Sällakapets i Lund Förhandlingar vol. 5 (1935) No. 5.

8. N. Hofreiter, Quadratische Zahlkörper ohne Euklidischen Algorithmus, Math. Ann. vol. 110 (1935) pp. 195-196.

9. J. F. Keston, Existence of a Euclidean algorithm in quadratic fields, Thesis, Yale University, 1935; cf. Bull. Amer. Math. Soc. vol. 41 (1935) p. 186.

10. H. Behrbohm and L. Rédei, Dern Euklidische Algorithmus in quadratischen Körpern, J. Reine Angew. Math. vol. 174 (1935) pp. 192-205. 
11. P. Erdös and Ch. Ko, Note on the Euclidean algorithm, J. London Math. Soc. vol. 13 (1938) pp. 3-8.

12. H. Heilbronn, On Euclid's algorithm in real quadratic fields, Proc. Cambridge Philos. Soc. vol. 34 (1938) pp. 521-526.

13. L. Schuster, Reelquadratische Zahlkörper ohne Euklidischen Algorithmus, Monatshefte für Mathematik und Physik vol. 47 (1938) pp. 117-127.

14. A. Brauer, On the non-existence of the Euclidean algorithm in certain quadratic number fields, Amer. J. Math. vol. 62 (1940) pp. 697-716.

15. L. Rédei, Zur Frage des Euklidischen Algorithmus in quadratischen Zahlkörpern, Math. Ann. vol. 118 (1942) pp. 588-608.

16. L. K. Hua and S. H. Min, On the distribution of quadratic non-residues and the Euclidean algorithm in real quadratic fields. II, Trans. Amer. Math. Soc. vol. 56 (1944) pp. 547-569.

17. L. K. Hua and W. T. Sheh, On the lack of an Euclidean algorithm in $R\left(61^{1 / 2}\right)$, Amer. J. Math. vol. 67 (1945) pp. 209-211.

18. L. Réidei, Über den Euklidischen Algorithmus in reellquadratischen Zahlkörpern, Matematiskai és Fizikai Lapok vol. 47 (1940) pp. 78-90.

19. H. Davenport, Indefinite binary quadratic forms, and Euclid's algorithm in real quadratic fields, To appear in Proc. London Math. Soc.

Ohio State University 